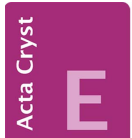
COMMUNICATIONS

ISSN 2056-9890

\section{Crystal structure of 3-(hydroxymethyl)- chromone}

\section{Yoshinobu Ishikawa}

School of Pharmaceutical Sciences, University of Shizuoka, 52-1 Yada, Suruga-ku, Shizuoka 422-8526, Japan. *Correspondence e-mail: ishi206@u-shizuoka-ken.ac.jp

Received 11 June 2015; accepted 16 June 2015

Edited by W. T. A. Harrison, University of Aberdeen, Scotland

In the title compound, $\mathrm{C}_{10} \mathrm{H}_{8} \mathrm{O}_{3}$ (systematic name 3-hydroxymethyl-4H-chromen-4-one), the fused-ring system is slightly puckered [dihedral angle between the rings $=3.84(11)^{\circ}$ ]. The hydroxy $\mathrm{O}$ atom deviates from the heterocyclic ring by 1.422 (1) $\AA$. In the crystal, inversion dimers linked by pairs of $\mathrm{O}-\mathrm{H} \cdots \mathrm{O}$ hydrogen bonds generate $R_{2}^{2}(12)$ loops. The dimers are linked by aromatic $\pi-\pi$ stacking [shortest centroidcentroid distance $=3.580(3) \AA]$, and $\mathrm{C}-\mathrm{H} \cdots \mathrm{O}$ hydrogen bonds, generating a three-dimensional network.

Keywords: crystal structure; chromone; hydrogen bonding; $\pi-\pi$ stacking.

CCDC reference: 1406927

\section{Related literature}

For the biological activities of related compounds, see: Sun et al. (2009); Helguera et al. (2013); Venkateswararao et al. (2014). For the synthesis of the title compound, see: ArayaMaturana et al. (2003).<smiles>O=c1c(CO)coc2ccccc12</smiles>

\section{Experimental}

\subsection{Crystal data}

$$
\begin{aligned}
& \mathrm{C}_{10} \mathrm{H}_{8} \mathrm{O}_{3} \\
& M_{r}=176.17 \\
& \text { Triclinic, } P \overline{1}
\end{aligned}
$$

$$
\begin{aligned}
& \alpha=94.48(6)^{\circ} \\
& \beta=108.27(5)^{\circ} \\
& \gamma=103.31(5)^{\circ} \\
& V=393.2(5) \AA^{3} \\
& Z=2
\end{aligned}
$$

$$
\begin{aligned}
& \text { Mo } K \alpha \text { radiation } \\
& \mu=0.11 \mathrm{~mm}^{-1} \\
& T=100 \mathrm{~K} \\
& 0.32 \times 0.32 \times 0.16 \mathrm{~mm}
\end{aligned}
$$

\subsection{Data collection}

Rigaku AFC-7R diffractometer 2219 measured reflections 1805 independent reflections 1537 reflections with $F^{2}>2.0 \sigma\left(F^{2}\right)$

\subsection{Refinement}

$R\left[F^{2}>2 \sigma\left(F^{2}\right)\right]=0.065$

$w R\left(F^{2}\right)=0.202$

$S=1.06$

1805 reflections
119 parameters

$\mathrm{H}$-atom parameters constrained

$\Delta \rho_{\max }=0.42 \mathrm{e}^{-3}$

$\Delta \rho_{\min }=-0.49 \mathrm{e} \AA^{-3}$

Table 1

Hydrogen-bond geometry $\left(\AA,^{\circ}\right)$.

\begin{tabular}{lllll}
\hline$D-\mathrm{H} \cdots A$ & $D-\mathrm{H}$ & $\mathrm{H} \cdots A$ & $D \cdots A$ & $D-\mathrm{H} \cdots A$ \\
\hline $\mathrm{O} 3-\mathrm{H} 8 \cdots \mathrm{O} 2^{\mathrm{i}}$ & 0.84 & 1.94 & $2.757(3)$ & 165 \\
$\mathrm{C} 1-\mathrm{H} 1 \cdots \mathrm{O} 2^{\text {ii }}$ & 0.95 & 2.58 & $3.283(4)$ & 131 \\
\hline
\end{tabular}

Symmetry codes: (i) $-x+2,-y+1,-z+1$; (ii) $x-1, y, z$.

Data collection: WinAFC Diffractometer Control Software (Rigaku, 1999); cell refinement: WinAFC Diffractometer Control Software; data reduction: WinAFC Diffractometer Control Software; program(s) used to solve structure: SIR2008 (Burla et al., 2007); program(s) used to refine structure: SHELXL97 (Sheldrick, 2008); molecular graphics: CrystalStructure (Rigaku, 2010); software used to prepare material for publication: CrystalStructure.

\title{
Acknowledgements
}

The University of Shizuoka is acknowledged for instrumental support.

Supporting information for this paper is available from the IUCr electronic archives (Reference: HB7444).

\section{References}

Araya-Maturana, R., Heredia-Moya, J., Pessoa-Mahana, H. \& Weiss-López, B. (2003). Synth. Commun. 33, 3225-3231.

Burla, M. C., Caliandro, R., Camalli, M., Carrozzini, B., Cascarano, G. L., De Caro, L., Giacovazzo, C., Polidori, G., Siliqi, D. \& Spagna, R. (2007). J. Appl. Cryst. 40, 609-613.

Helguera, A. M., Pérez-Garrido, A., Gaspar, A., Reis, J., Cagide, F., Vina, D., Cordeiro, M. N. \& Borges, F. (2013). Eur. J. Med. Chem. 59, 75-90.

Rigaku (1999). WinAFC Diffractometer Control Software. Rigaku Corporation, Tokyo, Japan.

Rigaku (2010). CrystalStructure. Rigaku Corporation, Tokyo, Japan.

Sheldrick, G. M. (2008). Acta Cryst. A64, 112-122.

Sun, W., Carroll, P. J., Soprano, D. R. \& Canney, D. J. (2009). Bioorg. Med. Chem. Lett. 19, 4339-4342.

Venkateswararao, E., Sharma, V. K., Manickam, M., Yun, J. \& Jung, S. H. (2014). Bioorg. Med. Chem. Lett. 24, 5256-5259. 


\section{supporting information}

Acta Cryst. (2015). E71, o495 [doi:10.1107/S2056989015011627]

\section{Crystal structure of 3-(hydroxymethyl)chromone}

\section{Yoshinobu Ishikawa}

\section{S1. Comment}

Many derivatives of the title compound (3-hydroxymethylchromone) are reported as retinoic acid receptor binders (Sun et al. (2009)), human monoamine oxidase inhibitors (Helguera et al. (2013)) and anti-proliferative agents

(Venkateswararao et al. (2014)).

The mean deviation of the least-square planes for the non-hydrogen atoms except hydroxy O3 atom is $0.0479 \AA$, and the largest deviation is 0.146 (2) $\AA$ for $\mathrm{C} 10$. These mean that these atoms are essentially coplanar (Fig.1). The dihedral angle of $\mathrm{C} 3-\mathrm{C} 2-\mathrm{C} 10-\mathrm{O} 3$ is 70.6 (2). In the crystal, the pyran rings are stacked [centroid-centroid distance between the pyran rings of the $4 H$-chromene units $=3.894(3) \AA]$, and $\mathrm{C}-\mathrm{H} \cdots \mathrm{O}$ hydrogen bonds are formed to give dimers running along the $c$ direction, as shown in Fig.2.

\section{S2. Experimental}

The title compound was synthesized from 3-formylchromone according to the literature method (Araya-Maturana et al. 2003). Colourless blocks were obtained by slow evaporation of an ethyl acetate solution of the title compound at room temperature.

\section{S3. Refinement}

All hydrogen atoms were placed in geometrical positions [C-H $0.95 \AA$ and $\mathrm{O}-\mathrm{H} 0.84 \AA$ ], and refined using a riding model with $U_{\text {iso }}(\mathrm{H})=1.2 U_{\text {eq }}$ of the parent atoms.

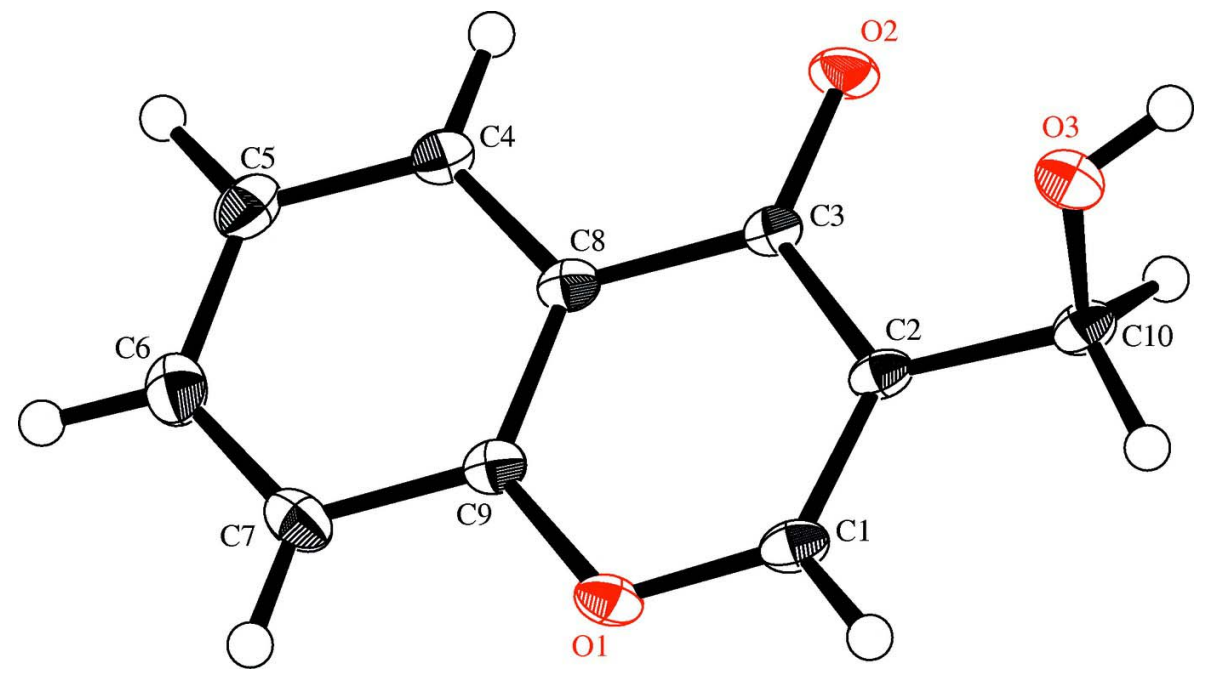




\section{Figure 1}

The molecular structure of the title compound, with displacement ellipsoids drawn at the $50 \%$ probability level.

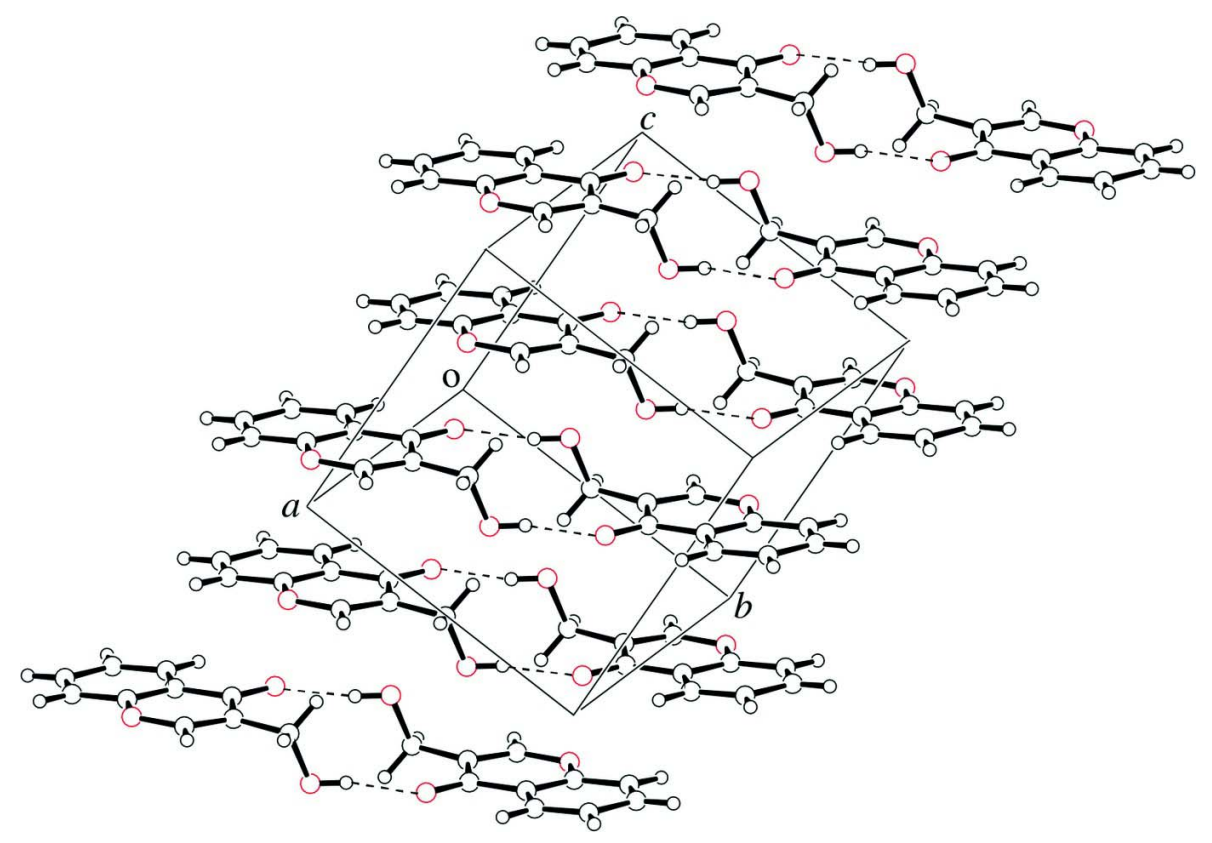

\section{Figure 2}

A view of the packing of the title compound. $\mathrm{O}-\mathrm{H} \cdots \mathrm{O}$ hydrogen bonds are represented as dashed lines.

\section{3-(Hydroxymethyl)-4H-chromen-4-one}

\section{Crystal data}

$\mathrm{C}_{10} \mathrm{H}_{8} \mathrm{O}_{3}$

$M_{r}=176.17$

Triclinic, $P \overline{1}$

Hall symbol: -P 1

$a=6.756(4) \AA$

$b=7.988(6) \AA$

$c=7.991(6) \AA$

$\alpha=94.48(6)^{\circ}$

$\beta=108.27(5)^{\circ}$

$\gamma=103.31(5)^{\circ}$

$V=393.2(5) \AA^{3}$

\section{Data collection}

Rigaku AFC-7R

diffractometer

$\omega-2 \theta$ scans

2219 measured reflections

1805 independent reflections

1537 reflections with $F^{2}>2.0 \sigma\left(F^{2}\right)$

$R_{\text {int }}=0.089$

\section{$Z=2$}

$F(000)=184.00$

$D_{\mathrm{x}}=1.488 \mathrm{Mg} \mathrm{m}^{-3}$

Mo $K \alpha$ radiation, $\lambda=0.71069 \AA$

Cell parameters from 25 reflections

$\theta=15.5-17.3^{\circ}$

$\mu=0.11 \mathrm{~mm}^{-1}$

$T=100 \mathrm{~K}$

Block, colorless

$0.32 \times 0.32 \times 0.16 \mathrm{~mm}$

$$
\begin{aligned}
& \theta_{\max }=27.5^{\circ} \\
& h=-4 \rightarrow 8 \\
& k=-10 \rightarrow 10 \\
& l=-10 \rightarrow 9
\end{aligned}
$$

3 standard reflections every 150 reflections intensity decay: $0.1 \%$ 


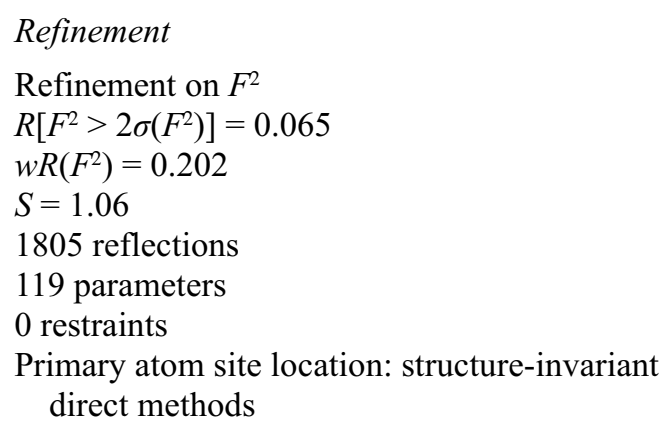

\section{Refinement}

$w R\left(F^{2}\right)=0.202$

$S=1.06$

805 reflections

direct methods

Special details

Refinement. Refinement was performed using all reflections. The weighted $R$-factor $(w R)$ and goodness of fit $(S)$ are based on $F^{2}$. $R$-factor (gt) are based on $F$. The threshold expression of $F^{2}>2.0 \sigma\left(F^{2}\right)$ is used only for calculating $R$-factor (gt).

Fractional atomic coordinates and isotropic or equivalent isotropic displacement parameters $\left(\AA^{2}\right)$

\begin{tabular}{lllll}
\hline & $x$ & $y$ & $z$ & $U_{\text {iso }} / U_{\text {eq }}$ \\
\hline O1 & $0.72779(19)$ & $1.01991(17)$ & $0.70911(18)$ & $0.0179(4)$ \\
O2 & $1.19522(19)$ & $0.80874(17)$ & $0.63213(18)$ & $0.0190(4)$ \\
O3 & $0.7925(2)$ & $0.48525(17)$ & $0.56522(17)$ & $0.0195(4)$ \\
C1 & $0.6785(3)$ & $0.8666(3)$ & $0.6006(3)$ & $0.0167(4)$ \\
C2 & $0.8224(3)$ & $0.7871(3)$ & $0.5707(3)$ & $0.0146(4)$ \\
C3 & $1.0527(3)$ & $0.8696(3)$ & $0.6575(3)$ & $0.0133(4)$ \\
C4 & $1.3221(3)$ & $1.1214(3)$ & $0.8824(3)$ & $0.0168(4)$ \\
C5 & $1.3681(3)$ & $1.2688(3)$ & $1.0053(3)$ & $0.0209(5)$ \\
C6 & $1.1985(3)$ & $1.3325(3)$ & $1.0269(3)$ & $0.0218(5)$ \\
C7 & $0.9868(3)$ & $1.2504(3)$ & $0.9255(3)$ & $0.0200(5)$ \\
C8 & $1.1070(3)$ & $1.0325(3)$ & $0.7810(3)$ & $0.0147(4)$ \\
C9 & $0.9416(3)$ & $1.0995(3)$ & $0.8033(3)$ & $0.0153(4)$ \\
C10 & $0.7477(3)$ & $0.6132(3)$ & $0.4539(3)$ & $0.0165(4)$ \\
H1 & 0.5294 & 0.8101 & 0.5403 & $0.0201^{*}$ \\
H2 & 1.4369 & 1.0798 & 0.8665 & $0.0202^{*}$ \\
H3 & 1.5139 & 1.3272 & 1.0751 & $0.0251^{*}$ \\
H4 & 1.2306 & 1.4333 & 1.1123 & $0.0262^{*}$ \\
H5 & 0.8729 & 1.2955 & 0.9382 & $0.0240^{*}$ \\
H6A & 0.5905 & 0.5855 & 0.3870 & $0.0198^{*}$ \\
H7B & 0.8247 & 0.6145 & 0.3669 & $0.0198^{*}$ \\
H8 & 0.8200 & 0.4052 & 0.5093 & $0.0234^{*}$ \\
& & & & \\
\hline
\end{tabular}

Atomic displacement parameters $\left(\AA^{2}\right)$

\begin{tabular}{lllllll}
\hline & $U^{11}$ & $U^{22}$ & $U^{33}$ & $U^{12}$ & $U^{13}$ & $U^{23}$ \\
\hline O1 & $0.0130(6)$ & $0.0202(7)$ & $0.0240(7)$ & $0.0070(5)$ & $0.0090(5)$ & $0.0043(5)$ \\
O2 & $0.0112(6)$ & $0.0225(7)$ & $0.0252(7)$ & $0.0067(5)$ & $0.0075(5)$ & $0.0032(6)$ \\
O3 & $0.0195(7)$ & $0.0188(7)$ & $0.0236(7)$ & $0.0069(5)$ & $0.0104(6)$ & $0.0052(5)$ \\
C1 & $0.0110(8)$ & $0.0212(9)$ & $0.0193(9)$ & $0.0041(7)$ & $0.0061(7)$ & $0.0073(7)$ \\
C2 & $0.0108(8)$ & $0.0202(9)$ & $0.0144(8)$ & $0.0046(7)$ & $0.0050(6)$ & $0.0067(7)$
\end{tabular}


supporting information

\begin{tabular}{lllllll} 
C3 & $0.0115(8)$ & $0.0168(9)$ & $0.0129(8)$ & $0.0043(6)$ & $0.0052(6)$ & $0.0053(6)$ \\
C4 & $0.0158(8)$ & $0.0179(9)$ & $0.0168(8)$ & $0.0054(7)$ & $0.0044(7)$ & $0.0050(7)$ \\
C5 & $0.0205(9)$ & $0.0212(10)$ & $0.0174(9)$ & $0.0040(7)$ & $0.0021(7)$ & $0.0055(7)$ \\
C6 & $0.0302(10)$ & $0.0184(9)$ & $0.0171(9)$ & $0.0071(8)$ & $0.0077(8)$ & $0.0042(7)$ \\
C7 & $0.0264(9)$ & $0.0198(9)$ & $0.0212(9)$ & $0.0113(8)$ & $0.0136(8)$ & $0.0068(7)$ \\
C8 & $0.0144(8)$ & $0.0179(9)$ & $0.0140(8)$ & $0.0055(7)$ & $0.0061(7)$ & $0.0071(7)$ \\
C9 & $0.0149(8)$ & $0.0191(9)$ & $0.0144(8)$ & $0.0055(7)$ & $0.0068(7)$ & $0.0069(7)$ \\
C10 & $0.0101(8)$ & $0.0204(9)$ & $0.0178(9)$ & $0.0026(7)$ & $0.0039(6)$ & $0.0038(7)$ \\
\hline
\end{tabular}

Geometric parameters $\left(\AA,{ }^{\circ}\right)$

\begin{tabular}{|c|c|c|c|}
\hline $\mathrm{O} 1-\mathrm{C} 1$ & $1.352(3)$ & $\mathrm{C} 6-\mathrm{C} 7$ & $1.375(3)$ \\
\hline $\mathrm{O} 1-\mathrm{C} 9$ & $1.371(2)$ & $\mathrm{C} 7-\mathrm{C} 9$ & $1.400(3)$ \\
\hline $\mathrm{O} 2-\mathrm{C} 3$ & $1.236(3)$ & $\mathrm{C} 8-\mathrm{C} 9$ & $1.396(3)$ \\
\hline $\mathrm{O} 3-\mathrm{C} 10$ & $1.429(3)$ & $\mathrm{O} 3-\mathrm{H} 8$ & 0.840 \\
\hline $\mathrm{C} 1-\mathrm{C} 2$ & $1.346(3)$ & $\mathrm{C} 1-\mathrm{H} 1$ & 0.950 \\
\hline $\mathrm{C} 2-\mathrm{C} 3$ & $1.455(3)$ & $\mathrm{C} 4-\mathrm{H} 2$ & 0.950 \\
\hline $\mathrm{C} 2-\mathrm{C} 10$ & $1.495(3)$ & $\mathrm{C} 5-\mathrm{H} 3$ & 0.950 \\
\hline $\mathrm{C} 3-\mathrm{C} 8$ & $1.468(3)$ & $\mathrm{C} 6-\mathrm{H} 4$ & 0.950 \\
\hline $\mathrm{C} 4-\mathrm{C} 5$ & $1.381(3)$ & $\mathrm{C} 7-\mathrm{H} 5$ & 0.950 \\
\hline $\mathrm{C} 4-\mathrm{C} 8$ & $1.404(3)$ & $\mathrm{C} 10-\mathrm{H} 6 \mathrm{~A}$ & 0.990 \\
\hline $\mathrm{C} 5-\mathrm{C} 6$ & $1.407(4)$ & $\mathrm{C} 10-\mathrm{H} 7 \mathrm{~B}$ & 0.990 \\
\hline $\mathrm{C} 1-\mathrm{O} 1-\mathrm{C} 9$ & $117.98(17)$ & $\mathrm{O} 3-\mathrm{C} 10-\mathrm{C} 2$ & $108.17(15)$ \\
\hline $\mathrm{O} 1-\mathrm{C} 1-\mathrm{C} 2$ & $125.63(15)$ & $\mathrm{C} 10-\mathrm{O} 3-\mathrm{H} 8$ & 109.472 \\
\hline $\mathrm{C} 1-\mathrm{C} 2-\mathrm{C} 3$ & $119.38(17)$ & $\mathrm{O} 1-\mathrm{C} 1-\mathrm{H} 1$ & 117.186 \\
\hline $\mathrm{C} 1-\mathrm{C} 2-\mathrm{C} 10$ & $120.66(15)$ & $\mathrm{C} 2-\mathrm{C} 1-\mathrm{H} 1$ & 117.188 \\
\hline $\mathrm{C} 3-\mathrm{C} 2-\mathrm{C} 10$ & $119.93(18)$ & $\mathrm{C} 5-\mathrm{C} 4-\mathrm{H} 2$ & 119.761 \\
\hline $\mathrm{O} 2-\mathrm{C} 3-\mathrm{C} 2$ & $123.48(17)$ & $\mathrm{C} 8-\mathrm{C} 4-\mathrm{H} 2$ & 119.765 \\
\hline $\mathrm{O} 2-\mathrm{C} 3-\mathrm{C} 8$ & $121.37(15)$ & $\mathrm{C} 4-\mathrm{C} 5-\mathrm{H} 3$ & 120.061 \\
\hline $\mathrm{C} 2-\mathrm{C} 3-\mathrm{C} 8$ & $115.15(18)$ & $\mathrm{C} 6-\mathrm{C} 5-\mathrm{H} 3$ & 120.064 \\
\hline $\mathrm{C} 5-\mathrm{C} 4-\mathrm{C} 8$ & $120.5(2)$ & $\mathrm{C} 5-\mathrm{C} 6-\mathrm{H} 4$ & 119.658 \\
\hline $\mathrm{C} 4-\mathrm{C} 5-\mathrm{C} 6$ & $119.88(16)$ & $\mathrm{C} 7-\mathrm{C} 6-\mathrm{H} 4$ & 119.653 \\
\hline $\mathrm{C} 5-\mathrm{C} 6-\mathrm{C} 7$ & $120.69(19)$ & $\mathrm{C} 6-\mathrm{C} 7-\mathrm{H} 5$ & 120.506 \\
\hline $\mathrm{C} 6-\mathrm{C} 7-\mathrm{C} 9$ & $119.0(3)$ & $\mathrm{C} 9-\mathrm{C} 7-\mathrm{H} 5$ & 120.503 \\
\hline $\mathrm{C} 3-\mathrm{C} 8-\mathrm{C} 4$ & $121.64(19)$ & $\mathrm{O} 3-\mathrm{C} 10-\mathrm{H} 6 \mathrm{~A}$ & 110.064 \\
\hline $\mathrm{C} 3-\mathrm{C} 8-\mathrm{C} 9$ & $119.77(15)$ & $\mathrm{O} 3-\mathrm{C} 10-\mathrm{H} 7 \mathrm{~B}$ & 110.063 \\
\hline $\mathrm{C} 4-\mathrm{C} 8-\mathrm{C} 9$ & $118.56(17)$ & $\mathrm{C} 2-\mathrm{C} 10-\mathrm{H} 6 \mathrm{~A}$ & 110.069 \\
\hline $\mathrm{O} 1-\mathrm{C} 9-\mathrm{C} 7$ & $116.70(19)$ & $\mathrm{C} 2-\mathrm{C} 10-\mathrm{H} 7 \mathrm{~B}$ & 110.064 \\
\hline $\mathrm{O} 1-\mathrm{C} 9-\mathrm{C} 8$ & $121.91(17)$ & $\mathrm{H} 6 \mathrm{~A}-\mathrm{C} 10-\mathrm{H} 7 \mathrm{~B}$ & 108.407 \\
\hline $\mathrm{C} 7-\mathrm{C} 9-\mathrm{C} 8$ & $121.38(16)$ & & \\
\hline $\mathrm{C} 1-\mathrm{O} 1-\mathrm{C} 9-\mathrm{C} 7$ & $-174.88(15)$ & $\mathrm{C} 5-\mathrm{C} 4-\mathrm{C} 8-\mathrm{C} 3$ & $-176.20(17)$ \\
\hline $\mathrm{C} 1-\mathrm{O} 1-\mathrm{C} 9-\mathrm{C} 8$ & $4.2(3)$ & $\mathrm{C} 5-\mathrm{C} 4-\mathrm{C} 8-\mathrm{C} 9$ & $1.9(3)$ \\
\hline $\mathrm{C} 9-\mathrm{O} 1-\mathrm{C} 1-\mathrm{C} 2$ & $-2.8(3)$ & $\mathrm{C} 8-\mathrm{C} 4-\mathrm{C} 5-\mathrm{C} 6$ & $-1.2(3)$ \\
\hline $\mathrm{C} 9-\mathrm{O} 1-\mathrm{C} 1-\mathrm{H} 1$ & 177.2 & $\mathrm{C} 8-\mathrm{C} 4-\mathrm{C} 5-\mathrm{H} 3$ & 178.8 \\
\hline $\mathrm{H} 8-\mathrm{O} 3-\mathrm{C} 10-\mathrm{C} 2$ & -148.1 & $\mathrm{H} 2-\mathrm{C} 4-\mathrm{C} 5-\mathrm{C} 6$ & 178.8 \\
\hline $\mathrm{H} 8-\mathrm{O} 3-\mathrm{C} 10-\mathrm{H} 6 \mathrm{~A}$ & 91.6 & $\mathrm{H} 2-\mathrm{C} 4-\mathrm{C} 5-\mathrm{H} 3$ & -1.2 \\
\hline
\end{tabular}




-27.8
$-1.0(3)$
$177.18(16)$
179.0
-2.8
$-177.19(17)$
$3.3(3)$
$-107.56(19)$
12.7
132.2
$70.6(2)$
-169.1
-49.7
$4.6(3)$
$-174.93(15)$
$-3.4(3)$
$178.52(16)$
$176.19(15)$
$-1.9(3)$

$\mathrm{H} 2-\mathrm{C} 4-\mathrm{C} 8-\mathrm{C} 3$

$\mathrm{H} 2-\mathrm{C} 4-\mathrm{C} 8-\mathrm{C} 9$

3.8

$\mathrm{C} 4-\mathrm{C} 5-\mathrm{C} 6-\mathrm{C} 7$

$\mathrm{C} 4-\mathrm{C} 5-\mathrm{C} 6-\mathrm{H} 4$

$\mathrm{H} 3-\mathrm{C} 5-\mathrm{C} 6-\mathrm{C} 7$

$\mathrm{H} 3-\mathrm{C} 5-\mathrm{C} 6-\mathrm{H} 4$

$\mathrm{C} 5-\mathrm{C} 6-\mathrm{C} 7-\mathrm{C} 9$

$\mathrm{C} 5-\mathrm{C} 6-\mathrm{C} 7-\mathrm{H} 5$

$\mathrm{H} 4-\mathrm{C} 6-\mathrm{C} 7-\mathrm{C} 9$

$\mathrm{H} 4-\mathrm{C} 6-\mathrm{C} 7-\mathrm{H} 5$

$\mathrm{C} 6-\mathrm{C} 7-\mathrm{C} 9-\mathrm{O} 1$

$\mathrm{C} 6-\mathrm{C} 7-\mathrm{C} 9-\mathrm{C} 8$

$\mathrm{H} 5-\mathrm{C} 7-\mathrm{C} 9-\mathrm{O} 1$

$\mathrm{H} 5-\mathrm{C} 7-\mathrm{C} 9-\mathrm{C} 8$

$\mathrm{C} 3-\mathrm{C} 8-\mathrm{C} 9-\mathrm{O} 1$

$\mathrm{C} 3-\mathrm{C} 8-\mathrm{C} 9-\mathrm{C} 7$

$\mathrm{C} 4-\mathrm{C} 8-\mathrm{C} 9-\mathrm{O} 1$

$\mathrm{C} 4-\mathrm{C} 8-\mathrm{C} 9-\mathrm{C} 7$
$-178.1$

$-0.7(3)$

179.3

179.3

$-0.7$

$1.6(3)$

$-178.4$

$-178.4$

1.6

$178.25(17)$

$-0.8(3)$

$-1.8$

179.2

$-1.8(3)$

$177.23(16)$

$-179.96(16)$

-0.9 (3)

Hydrogen-bond geometry $\left(A,{ }^{\circ}\right)$

\begin{tabular}{lllll}
\hline$D-\mathrm{H} \cdots A$ & $D-\mathrm{H}$ & $\mathrm{H} \cdots A$ & $D \cdots A$ & $D-\mathrm{H} \cdots A$ \\
\hline $\mathrm{O} 3-\mathrm{H} 8 \cdots \mathrm{O} 2^{\mathrm{i}}$ & 0.84 & 1.94 & $2.757(3)$ & 165 \\
$\mathrm{C} 1-\mathrm{H} 1 \cdots \mathrm{O} 2^{\mathrm{ii}}$ & 0.95 & 2.58 & $3.283(4)$ & 131 \\
\hline
\end{tabular}

Symmetry codes: (i) $-x+2,-y+1,-z+1$; (ii) $x-1, y, z$. 